\title{
Some Common Fixed Point Theorems Satisfying $(\psi-\varphi)$ Maps in Partial Metric Spaces
}

\author{
Reza Arab* \\ Department of Mathematics, Sari Branch, Islamic Azad University, Sari, Iran \\ *Corresponding author: mathreza.arab@iausari.ac.ir
}

Received February 06, 2015; Revised March 12, 2015; Accepted May 07, 2015

\begin{abstract}
In this paper, we present some coincidence and common fixed point results for infinite families of contractive maps satisfying a new class of pairs of generalized contractive type mappings defined in partial metric spaces. Our results extend and generalize many known results in the literature. Also, we introduce an example to support the validity of our results.
\end{abstract}

Keywords: partial metric, common fixed point, coincidence point, generalized contraction

Cite This Article: Reza Arab, "Some Common Fixed Point Theorems Satisfying $(\psi-\varphi)$ Maps in Partial Metric Spaces.” Turkish Journal of Analysis and Number Theory, vol. 3, no. 2 (2015): 53-60. doi: 10.12691/tjant3-2-4.

\section{Introduction and Preliminaries}

In 1994, in [8] Matthews introduced the notion of a partial metric space and proved the contraction principle of Banach in this new framework. The Existence of a fixed point for the contraction type mappings in partially metric spaces and its applications have been considered recently by many authors $[1,2,3,6,7,9,12,13,15]$. Consistent with $[4,8]$, the following de_nitions and results will be needed in the sequel.

Definition 1.1. [8] A partial metric on a nonempty set $X$ is a function $p: X \times X \rightarrow R^{+}$such that for all $x, y, z \in X:$

$\left(\mathrm{P}_{1}\right) \mathrm{x}=\mathrm{y} \Leftrightarrow \mathrm{p}(\mathrm{x}, \mathrm{x})=\mathrm{p}(\mathrm{x}, \mathrm{y})=\mathrm{p}(\mathrm{y}, \mathrm{y})$,

$\left(\mathrm{P}_{2}\right) \mathrm{p}(\mathrm{x}, \mathrm{x}) \leq \mathrm{p}(\mathrm{x}, \mathrm{y})$,

$\left(\mathrm{P}_{3}\right) \mathrm{p}(\mathrm{x}, \mathrm{y})=\mathrm{p}(\mathrm{y}, \mathrm{x})$,

$\left(\mathrm{P}_{4}\right) \mathrm{p}(\mathrm{x}, \mathrm{y}) \leq \mathrm{p}(\mathrm{x}, \mathrm{z})+\mathrm{p}(\mathrm{z}, \mathrm{y})-\mathrm{p}(\mathrm{z}, \mathrm{z})$.

A partial metric space is a pair $(X, p)$ such that $X$ is a nonempty set and $p$ is a partial metric on $X$.

It is clear that, if $p(x, y)=0$, then, we get $x=y$ from $\left(P_{1}\right)$ and $\left(P_{2}\right)$. But if $x=y, p(x, y)$ may be a positive number other than 0 .

Example 1.2. [8] Let a function $p: R^{+} \times R^{+} \rightarrow R^{+}$be defined by $p(x, y)=\max \{x, y\}$ for any $x, y \in R^{+}$. Then, $\left(R^{+}, p\right)$ is a partial metric space.

Example 1.3. [8] If $X=\{[a, b]: a, b \in R, a \leq b\}$, then $p$ : $X \times X \rightarrow R^{+}$de_ned by $p([a, b],[c, d])=\max \{b, d\}-$ $\min \{a, c\}$ is a partial metric on $X$.

If $p$ is a partial metric on $X$, then the function $p^{s}: X \times X$ $\rightarrow R^{+}$given by

$$
p^{s}(x, y)=2 p(x, y)-p(x, x)-p(y, y)
$$

is a metric on $X$.

Definition 1.4. $[8,10,11]$ Let $(X, p)$ be a partial metric space. Then (i) A sequence $\{x n\}$ in a partial metric space $(X, p)$ converges to a point $x \in X$ if and only if $p(x, x)=\lim _{n \rightarrow \infty} p\left(x, x_{n}\right)$.

(ii) A sequence $\left\{X_{n}\right\}$ in a partial metric space $(X, p)$ is called a Cauchy sequence if there exists (and is finite $\lim _{n, m \rightarrow \infty} p\left(x_{m}, x_{n}\right)$.

(iii) A partial metric space $(X, p)$ is said to be complete if every Cauchy sequence $\{\mathrm{xn}\}$ in $\mathrm{X}$ converges to a point $X$ $\in X$, that is $p(x, x)=\lim _{n, m \rightarrow \infty} p\left(x_{m}, x_{n}\right)$.

It is easy to see that, every closed subset of a complete partial metric space is complete.

Lemma 1.5. $[8,9,10]$ Let $(X, p)$ be a partial metric space. Then

(a) $\left\{x_{n}\right\}$ is a Cauchy sequence in $(X, p)$ if and only if it is a Cauchy sequence in the metric space $\left(X, p^{s}\right)$.

(b) A partial metric space $(X, p)$ is complete if and only if the metric space $\left(X, p^{s}\right)$ is complete. Further-more,

$$
\lim _{n \rightarrow \infty} p^{s}\left(x_{n}, x\right)=0
$$

if and only if

$$
p(x, x)=\lim _{n \rightarrow \infty} p\left(x, x_{n}\right)=\lim _{n, m \rightarrow \infty} p\left(x_{m}, x_{n}\right) .
$$

Lemma 1.6. [2] A mapping $f: X-\rightarrow X$ is said to be continuous at $a \in X$, if for every $\epsilon>0$, there exists $\delta>0$ such that $f(B(a, \delta)) \subset B(f(a), \epsilon)$.

The following result is easy to check.

Lemma 1.7. Let $(X, p)$ be a partial metric space. $T: X$ $\rightarrow X$ is continuous if and only if given a sequence $\left\{x_{n}\right\} \subseteq X$ and $x \in X$ such that $p(x, x)=\lim _{n \rightarrow \infty} p\left(x_{n}, x\right)$, then $p(T x, T x)=\lim _{n \rightarrow \infty} p\left(T x_{n}, T x\right)$.

Definition 1.8. Let $X$ be a set, $\mathrm{T}$ and $\mathrm{g}$ are selfmaps of $\mathrm{X}$. A point $\mathrm{X}$ in $\mathrm{X}$ is called a coincidence point of $\mathrm{T}$ and $\mathrm{g}$ 
if $\mathrm{Tx}=\mathrm{gx}$. We shall call $\mathrm{w}=\mathrm{Tx}=\mathrm{gx}$ a point of coincidence of $\mathrm{T}$ and $\mathrm{g}$.

Definition 1.9. [14] Let (X, p) be a partial metric space and $\mathrm{T}, \mathrm{g}: \mathrm{X} \rightarrow \mathrm{X}$ are mappings of $\mathrm{X}$ into itself. We say that the pair $\{\mathrm{T}, \mathrm{g}\}$ is partial compatible if the following conditions is held:

$\left(\mathrm{b}_{1}\right)$ if $\mathrm{p}(\mathrm{x}, \mathrm{x})=0$ then $\mathrm{p}(\mathrm{gx}, \mathrm{gx})=0$,

$\left(\mathrm{b}_{2}\right) \lim _{n \rightarrow \infty} p\left(T\left(g x_{n}\right), g\left(T x_{n}\right)\right)=0$ whenever $\left\{x_{n}\right\}$ is a sequence in $X$ such that Txn $\rightarrow t$ and $g x n \rightarrow t$ for some $t$ $\in X$.

Example 1.10. Let $X=[0, \infty)$ endowed with the usual partial metric $p$ de_ned by $p: X \times X \rightarrow X$ with $p(x, y)=$ $\max \{x, y\}$. Suppose $T, g: X \rightarrow X$ such that $T x=5 x^{3}$ and $g x=2 x^{3}$ for all $x \in X$. It is easy to check that the pair $\{T$, $g\}$ is partial compatible.

Matthews [8] obtained the following Banach fixedpoint theorem on complete partial metric spaces.

Theorem 1.11. (Matthews[8]) Let $f$ be a mapping of a complete dualistic partial metric space $(X, p)$ into itself such that there is a real number $c$ with $0 \leq c<1$, satisfying

$$
p(f(x), f(y)) \leq c p(x, y),
$$

for all $x, y \in X$, then $f$ has a unique fixed point.

Recently, I. Altun and A. Erduran [5] obtained the following nice result, which generalizes Theorem 1.11 of Matthews.

Theorem 1.12. [5] Let $(X, p)$ be a complete partial metric space and let $f: X \rightarrow X$ be a map such that

$$
p(f(x), f(y)) \leq \phi p(x, y),
$$

for all $x, y \in X$, where $\phi:[0, \infty) \rightarrow[0, \infty)$ is a continuous, non-decreasing function such that $\phi(t)<t$ for each $t>0$. Then $f$ has a unique fixed point.

Theorem 1.13. [3] Let $(X, p)$ be a complete partial metric space and let $F: X \rightarrow X$ be a map such that

$$
p(F x, F y) \leq \phi\left(\max \left\{\begin{array}{l}
p(x, y), p(x, F x), p(y, F y), \\
\frac{1}{2}[p(x, F y)+p(y, F x)]
\end{array}\right\}\right)
$$

for all $x, y \in X$, where $\phi:[0, \infty) \rightarrow[0, \infty)$ is continuous, nondecreasing function such that $\phi(t)<t$ for each $t>0$, then $F$ has a unique fixed point.

\section{Main Results}

In this section, we fixed the set of functions by $\psi, \varphi$ : $[0,+\infty) \rightarrow[0,+\infty)$ such that
(a) $\psi$ is increasing;
(b) $\varphi(t)<\psi(t)$ for each $t>0, \varphi(0)=\psi(0)=0$;
(c) $\varphi(t)$ and $\psi(t)$ are continuous functions.

Define $\Psi=\{(\psi, \varphi): \psi$ and $\varphi$ satisfy (a),(b) and (c) $\}$.

Now, we establish an existence of common fixed point of a family mappings satisfying contractive condition involving $\psi-\varphi$ functions in the setup of partial metric spaces. Our results generalize Theorems 1.11,1.12 and 1.13 .

Theorem 2.1. Let $(X, p)$ be a complete partial metric space. Suppose $\left\{T_{\alpha}: X \rightarrow X: \alpha \in I\right\}$ be a family of mappings and $g: X \rightarrow X$ be a self map. Also there exists $i_{0}$ $\in I$ such that $T_{i_{0}} \subseteq g(X), g X$ is closed in $(X, p)$ and assume that there exists $(\psi, \varphi) \in \Psi$ such that

$$
\psi\left(p\left(T_{i_{0}} x, T_{i} y\right)\right) \leq \varphi(M(x, y)),
$$

for all $x, y \in X$, where

$$
M(x, y)=\max \left\{\begin{array}{l}
p(g x, g y), p\left(g x, T_{i_{0}} x\right), p\left(g y, T_{i} y\right), \\
\frac{p\left(g x, T_{i} y\right)+p\left(g y, T_{i_{0}} x\right)}{2}
\end{array}\right\} .
$$

Then, there exists a unique $\mathrm{x} \in \mathrm{X}$ such that gx $=$ Tix for all $\mathrm{i} \in \mathrm{I}$, that is, $\mathrm{g}$ and $\left\{\mathrm{T}_{\mathrm{i}}\right.$ : $\left.\mathrm{i} \in \mathrm{I}\right\}$ have a unique coincidence point in $\mathrm{X}$. Moreover, any coincidence point of $g$ and $T_{i_{0}}$ is a coincidence point of $g$ and $\left\{T_{i}: i \in I\right\}$. Also, we have $\mathrm{p}(\mathrm{gx}, \mathrm{gx})=0$.

Proof. Let $x_{0}$ be an arbitrary point in $X$. Construct the sequence $\left\{x_{n}\right\}$ such that $T_{i_{0}}\left(x_{n}\right)=g\left(x_{n}+1\right)$ for each $n=0,1$, 2, .... which is possible since $T_{i_{0}} X \subseteq g X$. Now by (2.1), we have

$$
\psi\left(p\left(T_{i_{0}}\left(x_{n}\right), T_{i_{0}}\left(x_{n+1}\right)\right)\right) \leq \varphi\left(M\left(x_{n}, x_{n+1}\right)\right),
$$

where

$$
\begin{aligned}
& M\left(x_{n}, x_{n+1}\right) \\
& =\max \left\{\begin{array}{l}
p\left(g\left(x_{n}\right), g\left(x_{n+1}\right)\right), p\left(g\left(x_{n}\right), T_{\alpha_{0}}\left(x_{n}\right)\right), \\
p\left(g\left(x_{n+1}\right), T_{i_{0}}\left(x_{n+1}\right)\right), \\
\frac{p\left(g\left(x_{n}\right), T_{i_{0}}\left(x_{n+1}\right)\right)+p\left(g\left(x_{n+1}\right), T_{i_{0}}\left(x_{n}\right)\right)}{2}
\end{array}\right\}
\end{aligned}
$$

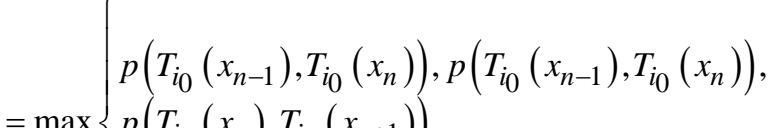

$$
\begin{aligned}
& =\max \left\{p\left(T_{i_{0}}\left(x_{n}\right), T_{i_{0}}\left(x_{n+1}\right)\right)\right. \text {, } \\
& \frac{p\left(T_{i_{0}}\left(x_{n-1}\right), T_{i_{0}}\left(x_{n+1}\right)\right)+p\left(T_{i_{0}}\left(x_{n}\right), T_{i_{0}}\left(x_{n}\right)\right)}{p\left(T_{i_{0}}\left(x_{n-1}\right), T_{i_{0}}\left(x_{n}\right)\right), p\left(T_{i_{0}}\left(x_{n}\right), T_{i_{0}}\left(x_{n+1}\right)\right),} \\
& =\max \left\{\frac{p\left(T_{i_{0}}\left(x_{n-1}\right), T_{i_{0}}\left(x_{n+1}\right)\right)+p\left(T_{i_{0}}\left(x_{n}\right), T_{i_{0}}\left(x_{n}\right)\right)}{2}\right\} .
\end{aligned}
$$

By $\left(p_{4}\right)$, we have

$$
\begin{aligned}
& \frac{p\left(T_{i_{0}}\left(x_{n-1}\right), T_{i_{0}}\left(x_{n+1}\right)\right)+p\left(T_{i_{0}}\left(x_{n}\right), T_{i_{0}}\left(x_{n}\right)\right)}{2} \\
& \leq \frac{p\left(T_{i_{0}}\left(x_{n-1}\right), T_{i_{0}}\left(x_{n}\right)\right)+p\left(T_{i_{0}}\left(x_{n}\right), T_{i_{0}}\left(x_{n+1}\right)\right)}{2} \\
& \leq \max \left\{p\left(T_{i_{0}}\left(x_{n-1}\right), T_{i_{0}}\left(x_{n}\right)\right), p\left(T_{i_{0}}\left(x_{n}\right), T_{i_{0}}\left(x_{n+1}\right)\right)\right\}
\end{aligned}
$$

Therefore,

$$
M\left(x_{n}, x_{n+1}\right)=\max \left\{\begin{array}{l}
p\left(T_{i_{0}}\left(x_{n-1}\right), T_{i_{0}}\left(x_{n}\right)\right), \\
p\left(T_{i_{0}}\left(x_{n}\right), T_{i_{0}}\left(x_{n+1}\right)\right)
\end{array}\right\} .
$$

If we suppose that

$$
M\left(x_{n}, x_{n+1}\right)=p\left(T_{i_{0}}\left(x_{n}\right), T_{i_{0}}\left(x_{n+1}\right)\right)
$$


and $T_{i_{0}}\left(x_{n}\right)=T_{i_{0}}\left(x_{n+1}\right)$ for some $n$, then by $T_{i_{0}}\left(x_{n}\right)=$ $g\left(x_{n+1}\right), T_{i_{0}}\left(x_{n+1}\right)=g\left(x_{n+1}\right)$, that is, $T_{i_{0}}$ and $g$ have a coincidence at $x=x_{n+1}$, and so the proof is completed, otherwise if $p\left(T_{i_{0}}\left(x_{n}\right), T_{i_{0}}\left(x_{n+1}\right)\right)>0$, then

$$
\begin{aligned}
\psi\left(p\left(T_{i_{0}}\left(x_{n}\right), T_{i_{0}}\left(x_{n+1}\right)\right)\right) & \leq \varphi\left(p\left(T_{i_{0}}\left(x_{n}\right), T_{i_{0}}\left(x_{n+1}\right)\right)\right) \\
& <\psi\left(p\left(T_{i_{0}}\left(x_{n}\right), T_{i_{0}}\left(x_{n+1}\right)\right)\right) .
\end{aligned}
$$

It is impossible.

If $M\left(x_{n}, x_{n+1}\right)=p\left(T_{i_{0}}\left(x_{n-1}\right), T_{i_{0}}\left(x_{n}\right)\right)$, we get

$$
\begin{aligned}
\psi\left(p\left(T_{i_{0}}\left(x_{n}\right), T_{i_{0}}\left(x_{n+1}\right)\right)\right) & \leq \varphi\left(p\left(T_{i_{0}}\left(x_{n-1}\right), T_{i_{0}}\left(x_{n}\right)\right)\right) \\
& <\psi\left(p\left(T_{i_{0}}\left(x_{n-1}\right), T_{i_{0}}\left(x_{n}\right)\right)\right) .
\end{aligned}
$$

If $T_{i_{0}}\left(x_{n-1}\right)=T_{i_{0}}\left(x_{n}\right)$ for some $n$, then by $T_{i_{0}}\left(x_{n-1}\right)=g\left(x_{n}\right), T_{i_{0}}\left(x_{n}\right)=g\left(x_{n}\right)$, that is, $T_{i_{0}}$ and $g$ have a coincidence at $x=x_{n}$, and so the proof is completed. Since we suppose that $p\left(T_{i_{0}}\left(x_{n-1}\right), T_{i_{0}}\left(x_{n}\right)\right)>0$ and by the monotone property of $\psi$ function, we have $p\left(T_{i_{0}}\left(x_{n}\right), T_{i_{0}}\left(x_{n+1}\right)\right)<p\left(T_{i_{0}}\left(x_{n-1}\right), T_{i_{0}}\left(x_{n}\right)\right)$. Therefore, the sequence $p\left(T_{i_{0}}\left(x_{n}\right), T_{i_{0}}\left(x_{n+1}\right)\right)$ of real numbers is monotone decreasing. Hence there exists a real number $r \geq 0$ such that,

$$
\lim _{n \rightarrow \infty} p\left(T_{i_{0}}\left(x_{n}\right), T_{i_{0}}\left(x_{n+1}\right)\right)=r
$$

We claim that $r=0$. On the contrary, assume that

$$
\lim _{n \rightarrow \infty} p\left(T_{i_{0}}\left(x_{n}\right), T_{i_{0}}\left(x_{n+1}\right)\right)=r>0 .
$$

Since $\psi$ and $\varphi$ are continuous then from (2.4) and (2.5), we have

$$
\begin{aligned}
& \psi(r)=\lim _{n \rightarrow \infty} \psi\left(p\left(T_{i_{0}}\left(x_{n}\right), T_{i_{0}}\left(x_{n+1}\right)\right)\right) \\
& =\lim _{n \rightarrow \infty} \varphi\left(p\left(T_{i_{0}}\left(x_{n-1}\right), T_{i_{0}}\left(x_{n}\right)\right)\right)=\varphi(r),
\end{aligned}
$$

and so $r=0$, a contradiction. Thus

$$
\lim _{n \rightarrow \infty} p\left(T_{i_{0}}\left(x_{n}\right), T_{i_{0}}\left(x_{n+1}\right)\right)=0 .
$$

From $p\left(T_{i_{0}}\left(x_{n}\right)\right.$, Ti0 $\left.\left(x_{n}\right)\right), p\left(T_{i_{0}}\left(x_{n+1}\right), T_{i_{0}}\left(x_{n+1}\right)\right) \leq$ $p\left(T_{i_{0}}\left(x_{n}\right), T_{i_{0}}\left(x_{n+1}\right)\right)$ and (2.6), we have

$$
\begin{aligned}
& \lim _{n \rightarrow \infty} p\left(T_{i_{0}}\left(x_{n}\right), T_{i_{0}}\left(x_{n}\right)\right) \\
& =\lim _{n \rightarrow \infty} p\left(T_{i_{0}}\left(x_{n+1}\right), T_{i_{0}}\left(x_{n+1}\right)\right)=0 .
\end{aligned}
$$

From (2.6), (2.7) and (1.1), we have

$$
\lim _{n \rightarrow \infty} p^{s}\left(T_{i_{0}}\left(x_{n}\right), T_{i_{0}}\left(x_{n+1}\right)\right)=0 .
$$

Next, we claim that $\left\{T_{i_{0}} x_{n}\right\}$ is a Cauchy sequence in the metric space $\left(X, p^{s}\right)$. Assume the contrary. Then there exists an $\epsilon>0$ and subsequences $\left\{x_{n(k)}\right\}$ and $\left\{x_{m(k)}\right\}$ of $\left\{x_{n}\right\}$ with $n(k)>m(k)>k$ such that $p\left(T_{i_{0}}\left(x_{n(k)}\right), T_{i_{0}}\left(x_{m(k)}\right)\right) \geq \epsilon$ and $p\left(T_{i_{0}}\left(x_{n(k)-1}\right), T_{i_{0}}\left(x_{m(k)}\right)\right)<\epsilon$.

Then we have

$$
\begin{aligned}
\epsilon & \leq p\left(T_{i_{0}}\left(x_{n(k)}\right), T_{i_{0}}\left(x_{m(k)}\right)\right) \\
\leq & p\left(T_{i_{0}}\left(x_{n(k)}\right), T_{i_{0}}\left(x_{n(k)-1}\right)\right) \\
& +p\left(T_{i_{0}}\left(x_{n(k)-1}\right), T_{i_{0}}\left(x_{m(k)}\right)\right) \\
& -p\left(T_{i_{0}}\left(x_{n(k)-1}\right), T_{i_{0}}\left(x_{n(k)-1}\right)\right) \\
< & p\left(T_{i_{0}}\left(x_{n(k)}\right), T_{i_{0}}\left(x_{n(k)-1}\right)\right)+\epsilon \\
& -p\left(T_{i_{0}}\left(x_{n(k)-1}\right), T_{i_{0}}\left(x_{n(k)-1}\right)\right),
\end{aligned}
$$

Taking $k \rightarrow \infty$ in (2.9) and using (2.6) and (2.7) we get

$$
\begin{aligned}
& \lim _{k \rightarrow \infty} p\left(T_{i_{0}}\left(x_{n(k)}\right), T_{i_{0}}\left(x_{m(k)}\right)\right) \\
& =\lim _{k \rightarrow \infty} p\left(T_{i_{0}}\left(x_{n(k)-1}\right), T_{i_{0}}\left(x_{m(k)}\right)\right)=\epsilon .
\end{aligned}
$$

Thus from the definition of $p$ we have

$$
\begin{aligned}
& p\left(T_{i_{0}}\left(x_{m(k)}\right), T_{i_{0}}\left(x_{n(k)}\right)\right) \\
& \leq p\left(T_{i_{0}}\left(x_{m(k)}\right), T_{i_{0}}\left(x_{m(k)-1}\right)\right) \\
& +p\left(T_{i_{0}}\left(x_{m(k)-1}\right), T_{i_{0}}\left(x_{n(k)-1}\right)\right) \\
& +p\left(T_{i_{0}}\left(x_{n(k)-1}\right), T_{i_{0}}\left(x_{n(k)}\right)\right) \\
& -p\left(T_{i_{0}}\left(x_{m(k)-1}\right), T_{i_{0}}\left(x_{m(k)-1}\right)\right) \\
& -p\left(T_{i_{0}}\left(x_{n(k)-1}\right), T_{i_{0}}\left(x_{n(k)-1}\right)\right) \text {, } \\
& p\left(T_{i_{0}}\left(x_{m(k)-1}\right), T_{i_{0}}\left(x_{n(k)-1}\right)\right) \\
& \leq p\left(T_{i_{0}}\left(x_{m(k)-1}\right), T_{i_{0}}\left(x_{m(k)}\right)\right) \\
& +p\left(T_{i_{0}}\left(x_{m(k)}\right), T_{i_{0}}\left(x_{n(k)}\right)\right) \\
& +p\left(T_{i_{0}}\left(x_{n(k)}\right), T_{i_{0}}\left(x_{n(k)-1}\right)\right) \\
& =p\left(T_{i_{0}}\left(x_{m(k)}\right), T_{i_{0}}\left(x_{m(k)}\right)\right) \\
& -p\left(T_{i_{0}}\left(x_{n(k)}\right), T_{i_{0}}\left(x_{n(k)}\right)\right) \text {. }
\end{aligned}
$$

Taking $k \rightarrow \infty$ in (2.11) and (2.12) and using (2.6), (2.7) and (2.10) we get

$$
\begin{aligned}
& \lim _{k \rightarrow \infty} p\left(T_{i_{0}}\left(x_{n(k)}\right), T_{i_{0}}\left(x_{m(k)}\right)\right) \\
& =\lim _{k \rightarrow \infty} p\left(T_{i_{0}}\left(x_{n(k)-1}\right), T_{i_{0}}\left(x_{m(k)-1}\right)\right)=\epsilon .
\end{aligned}
$$

Also,

$$
\begin{aligned}
& \epsilon \leq p\left(T_{i_{0}}\left(x_{n(k)}\right), T_{i_{0}}\left(x_{m(k)}\right)\right) \\
& \leq p\left(T_{i_{0}}\left(x_{n(k)}\right), T_{i_{0}}\left(x_{m(k)-1}\right)\right) \\
& +p\left(T_{i_{0}}\left(x_{m(k)-1}\right), T_{i_{0}}\left(x_{m(k)}\right)\right) \\
& -p\left(T_{i_{0}}\left(x_{m(k)-1}\right), T_{i_{0}}\left(x_{m(k)-1}\right)\right)
\end{aligned}
$$




$$
\begin{aligned}
& \leq p\left(T_{i_{0}}\left(x_{n(k)}\right), T_{i_{0}}\left(x_{n(k)-1}\right)\right) \\
& +p\left(T_{i_{0}}\left(x_{n(k)-1}\right), T_{i_{0}}\left(x_{m(k)-1}\right)\right) \\
& +p\left(T_{i_{0}}\left(x_{m(k)-1}\right), T_{i_{0}}\left(x_{m(k)}\right)\right) \\
& -p\left(T_{i_{0}}\left(x_{n(k)-1}\right), T_{i_{0}}\left(x_{n(k)-1}\right)\right) \\
& -p\left(T_{i_{0}}\left(x_{m(k)-1}\right), T_{i_{0}}\left(x_{m(k)-1}\right)\right) .
\end{aligned}
$$

Taking $k \rightarrow \infty$ in (2.14) and using (2.6), (2.7) and (2.13) we get

$$
\lim _{k \rightarrow \infty} p\left(T_{i_{0}}\left(x_{n(k)}\right), T_{i_{0}}\left(x_{m(k)-1}\right)\right)=\epsilon .
$$

Now using inequality (2.1), we have

$$
\begin{aligned}
& \psi(\epsilon) \leq \psi p\left(T_{i_{0}}\left(x_{n(k)}\right), T_{i_{0}}\left(x_{m(k)}\right)\right) \\
& \leq \varphi\left(M\left(x_{n(k)}, x_{m(k)}\right)\right),
\end{aligned}
$$

where

$$
\begin{aligned}
& M\left(x_{n(k)}, x_{m(k)}\right) \\
& =\max \left\{\begin{array}{l}
p\left(g\left(x_{n(k)}\right), g\left(x_{m(k)}\right)\right), \\
p\left(g\left(x_{n(k)}\right), T_{\alpha_{0}}\left(x_{n(k)}\right)\right), \\
p\left(g\left(x_{m(k)}\right), T_{i_{0}}\left(x_{m(k)}\right)\right), \\
{\left[\begin{array}{l}
\left.p\left(g\left(x_{n(k)}\right), T_{i_{0}}\left(x_{m(k)}\right)\right)\right) \\
+p\left(g\left(x_{m}\right)\right.
\end{array}\right.}
\end{array}\right. \\
& \frac{\left[+p\left(g\left(x_{m(k)}\right), T_{i_{0}}\left(x_{n(k)}\right)\right)\right]}{2} \\
& p\left(T_{i_{0}}\left(x_{n(k)-1}\right), T_{i_{0}}\left(x_{m(k)-1}\right)\right), \\
& p\left(T_{i_{0}}\left(x_{n(k)-1}\right), T_{i_{0}}\left(x_{n(k)}\right)\right) \text {, } \\
& =\max \left\{p\left(T_{i_{0}}\left(x_{m(k)-1}\right), T_{i_{0}}\left(x_{m(k)}\right)\right),\right. \\
& p\left(T_{i_{0}}\left(x_{n(k)-1}\right), T_{i_{0}}\left(x_{m(k)}\right)\right) \\
& \left.\frac{\left[+p\left(T_{i_{0}}\left(x_{m(k)-1}\right), T_{i_{0}}\left(x_{n(k)}\right)\right)\right]}{2}\right]
\end{aligned}
$$

Letting $k \rightarrow \infty$ in the above inequality and using (2.6),(2.10), (2.13) and (2.15), we obtain

$$
\lim _{k \rightarrow \infty} M\left(x_{n(k)}, x_{m(k)}\right)=\max \left\{\epsilon, 0,0, \frac{\epsilon+\epsilon}{2}\right\}=\epsilon .
$$

As $k \rightarrow \infty$, inequality (2.16) becomes,

$$
\psi(\epsilon) \leq \varphi(\epsilon)
$$

which is a contradiction by virtue of a property of $\varphi$.

Then, we deduce that $\left\{T_{i_{0}}\left(x_{n}\right)\right\}$ is a cauchy sequence in $\left(X, p^{s}\right)$. Hence, we have

$$
\lim _{m, n \rightarrow \infty} p^{s}\left(T_{i_{0}}\left(x_{m}\right), T_{i_{0}}\left(x_{n}\right)\right)=0 .
$$

Now, from the definition of $p^{s}$ and from (2.7), we have

$$
\lim _{m, n \rightarrow \infty} p\left(T_{i_{0}}\left(x_{m}\right), T_{i_{0}}\left(x_{n}\right)\right)=0 .
$$

Since $X$ is a complete partial metric space, then, from lemma 1.5, the sequence $\left\{T_{i_{0}}\left(x_{n}\right)\right\}$ converges in the metric space $\left(X, p^{s}\right)$, so there exist $z$ in $X$ such that

$$
\lim _{n \rightarrow \infty} p^{s}\left(T_{i_{0}}\left(x_{n}\right), z\right)=\lim _{n \rightarrow \infty} p^{s}\left(g x_{n+1}, z\right)=0 .
$$

Again, from Lemma 1.5, we get

$$
\begin{aligned}
& p(z, z)=\lim _{n \rightarrow \infty} p\left(T_{i_{0}}\left(x_{n}\right), z\right) \\
& =\lim _{n \rightarrow \infty} p\left(g x_{n+1}, z\right)=\lim _{n, m \rightarrow \infty} p\left(T_{\alpha_{0}}\left(x_{n}\right), T_{i_{0}}\left(x_{m}\right)\right) .
\end{aligned}
$$

But, from (2.17) and (2.18) we have

$$
p(z, z)=\lim _{n \rightarrow \infty} p\left(T_{i_{0}}\left(x_{n}\right), z\right)=\lim _{n \rightarrow \infty} p\left(g x_{n+1}, z\right)=0 .
$$

Since $\left\{T_{i_{0}}\left(x_{n}\right)\right\} \subseteq g X$ and $g X$ is closed, there exists $x \in X$ such that $z=g x$. Now, we claim that $x$ is a coincidence point of Ti0 and $g$. We have

$$
\begin{aligned}
& p\left(g x, T_{i_{0}}(x)\right) \\
& \leq p\left(g x, g x_{n+1}\right)+p\left(g x_{n+1}, T_{i_{0}}(x)\right)-p\left(g x_{n+1}, g x_{n+1}\right) \\
& =p\left(z, g x_{n+1}\right)+p\left(T_{i_{0}}\left(x_{n}\right), T_{i_{0}}(x)\right)-p\left(g x_{n+1}, g x_{n+1}\right) .
\end{aligned}
$$

On taking limit as $n \rightarrow \infty$ in the above inequality, we have

$$
p\left(g x, T_{i_{0}}(x)\right) \leq \lim _{n \rightarrow \infty} p\left(T_{i_{0}}\left(x_{n}\right), T_{i_{0}}(x)\right),
$$

By property of $\psi$ and using (2.20), we have

$$
\begin{aligned}
& \psi\left(p\left(g x, T_{i_{0}}(x)\right)\right) \leq \lim _{n \rightarrow \infty} \psi\left(p\left(T_{i_{0}}\left(x_{n}\right), T_{i_{0}}(x)\right)\right) \\
& \leq \lim _{n \rightarrow \infty} \varphi\left(M\left(x_{n}, x\right)\right)=\varphi\left(p\left(g x, T_{i_{0}}(x)\right)\right) .
\end{aligned}
$$

Indeed,

$$
\begin{aligned}
& p\left(g x, T_{i_{0}}(x)\right) \leq M\left(x_{n}, x\right) \\
& =\max \left\{\begin{array}{l}
p\left(g x_{n}, g x\right), p\left(g x_{n}, T_{i_{0}}\left(x_{n}\right)\right), p\left(g x, T_{i_{0}}(x)\right), \\
\frac{p\left(g x_{n}, T_{i_{0}}(x)\right)+p\left(g x, T_{i_{0}}\left(x_{n}\right)\right)}{2} \\
\leq \max \left\{\begin{array}{l}
p\left(g x_{n}, g x\right), p\left(g x_{n}, T_{i_{0}}\left(x_{n}\right)\right), p\left(g x, T_{i_{0}}(x)\right), \\
{\left[\begin{array}{l}
p\left(g x_{n}, g x\right)+p\left(g x, T_{i_{0}}(x)\right) \\
-p(g x, g x)+p\left(g x, T_{i_{0}}\left(x_{n}\right)\right)
\end{array}\right]} \\
\frac{2}{2}
\end{array}\right\}
\end{array}\right.
\end{aligned}
$$

we deduce, taking limit as $n \rightarrow \infty$, that

$$
\lim _{n \rightarrow \infty} M\left(x_{n}, x\right)=p\left(g x, T_{i_{0}}(x)\right) .
$$

By monotone non-decreasing property of $\psi$ and (2.21) we have $p\left(g x, T_{i_{0}}(x)\right)=0$, which implies that $T_{i_{0}}(x)=g x$, that is, $x$ is a coincidence point of $T_{i_{0}}$ and $g$.

Now suppose that $x \in X$ is a coincidence point of $T_{i_{0}}$ and $g$. Then for any $i \in I$, from (2.1), we have

$$
\psi p\left(g x, T_{i}(x)\right)=\psi\left(p T_{i_{0}}(x), T_{i}(x)\right) \leq \varphi(M(x, x))
$$


where

$$
\begin{aligned}
& M(x, x)=\max \left\{\begin{array}{l}
p(g(x), g(x)), p\left(g(x), T_{i_{0}}(x)\right), \\
p\left(g(x), T_{i}(x)\right) \\
\frac{p\left(g(x), T_{i}(x)\right)+p\left(g(x), T_{i_{0}}(x)\right)}{2}
\end{array}\right\} \\
& =p\left(g(x), T_{i}(x)\right) .
\end{aligned}
$$

So,

$$
\psi p\left(g x, T_{i}(x)\right) \leq \varphi p\left(g(x), T_{i}(x)\right),
$$

therefore $p\left(g x, T_{i}(x)\right)=0$, which implies that $T_{i}(x)=g x$, that is, $x$ is a coincidence point of $\left\{T_{i}: i \in I\right\}$ and $g$.

Assume that $z, y$ are coincidence points of $\left\{T_{i}: i \in I\right\}$ and $g$ in $X$ such that $y \neq z$. Then there exist $t_{1}, t_{2}$ in $X$ such that, for all $i \in I, T_{i}\left(t_{1}\right)=g\left(t_{1}\right)=z$ and $T_{i}\left(t_{2}\right)=g\left(t_{2}\right)=y$. Using (2.1), we have

$$
\psi\left(p\left(g t_{1}, g t_{2}\right)\right)=\psi\left(p\left(T_{i_{0}}\left(t_{1}\right), T_{i}\left(t_{2}\right)\right)\right) \leq \varphi\left(M\left(t_{1}, t_{2}\right)\right)
$$

where

$$
\begin{aligned}
& M\left(t_{1}, t_{2}\right) \\
& =\max \left\{\begin{array}{l}
p\left(g t_{1}, g t_{2}\right), p\left(g t_{1}, T_{i_{0}}\left(t_{1}\right)\right), p\left(g t_{2}, T_{i}\left(t_{2}\right)\right) \\
\frac{p\left(g t_{1}, T_{i}\left(t_{2}\right)\right)+p\left(g t_{2}, T_{i_{0}}\left(t_{1}\right)\right)}{2}
\end{array}\right\} \\
& =p\left(g t_{1}, g t_{2}\right) .
\end{aligned}
$$

So

$$
\psi\left(p\left(g t_{1}, g t_{2}\right)\right) \leq \varphi\left(p\left(g t_{1}, g t_{2}\right)\right)<\psi\left(p\left(g t_{1}, g t_{2}\right)\right),
$$

which is a contradiction which proves the uniqueness of point of coincidence.

Example 2.2. Let $X=[0,1]$ and $p(x, y)=\max \{x, y\}$, then it is clear that $(X, p)$ is a complete partial metric space. Let $I=\{1,2,3, \ldots\}, i 0=1$ and for every $i \in I, T i, g: X \rightarrow$ $X$, and $\psi, \varphi:[0, \infty) \rightarrow[0, \infty)$ be given by $T_{i}(x)=\frac{x^{3}}{(2 i+1)(x+3)}, g x=\frac{x^{2}}{2 x+6}, \psi(t)=2 t, \varphi(t)=\frac{4 t}{3}$.

Clearly $(\psi, \varphi) \in \Psi$. We show that condition (2.1) is satisfied.

$$
\begin{aligned}
& \text { If } x, y \in X \text {, then we have } \\
& \psi p\left(T_{i_{0}}(x), T_{i}(y)\right)=\psi\left(p\left(T_{1}(x), T_{i}(y)\right)\right) \\
& =\psi\left(\max \left\{\frac{x^{3}}{3 x+9}, \frac{y^{3}}{(2 i+1)(3 y+9)}\right\}\right) \\
& \leq 2 \max \max \left\{\frac{x^{3}}{3 x+9}, \frac{y^{3}}{(2 i+1)(3 y+9)}\right\} \\
& \leq \frac{2}{3} \max \left\{\frac{x^{3}}{x+3}, \frac{y^{3}}{(y+3)}\right\}=\frac{4}{3} p(g x, g x) \\
& \leq \frac{4}{3} \max \left\{\begin{array}{l}
p(g x, g x), p\left(g x, T_{1}(x)\right), p\left(g y, T_{i}(y)\right), \\
\frac{p\left(g x, T_{i}(y)\right), p\left(g y, T_{1}(x)\right)}{2}
\end{array}\right\} \\
& =\varphi(M(x, y)) \text {. }
\end{aligned}
$$

Note that, $\left\{T_{i}\right\}$ and $g$ satisfy all the conditions given in Theorem 2.1. Moreover, 0 is a unique common fixed point of $\left\{T_{i}\right\}$ and $g$.
As immediate consequences of Theorem 2.1 are the following fixed point results.

Corollary 2.3. Let $(X, p)$ be a complete partial metric space. Suppose $T_{1}, T_{2}, g: X \rightarrow X$ be three self mappings such that $T_{1}(x) \subseteq g(X), g X$ is closed in $(X, p)$ and for all $x, y \in X$

$$
\psi\left(p\left(T_{1} x, T_{2} y\right)\right) \leq \varphi(M(x, y))
$$

where

$$
M(x, y)=\max \left\{\begin{array}{l}
p(g x, g y), p\left(g x, T_{1} x\right), p\left(g y, T_{2} y\right), \\
\frac{p\left(g x, T_{2} y\right), p\left(g y, T_{1} x\right)}{2}
\end{array}\right\},
$$

and $(\psi, \varphi) \in \Psi$. Then, there exists a unique $\mathrm{x} \in \mathrm{X}$ such that $\mathrm{gx}=\mathrm{T}_{1} \mathrm{x}=\mathrm{T}_{2} \mathrm{x}$. Moreover, we have $\mathrm{p}(\mathrm{gx}, \mathrm{gx})=0$.

Proof. Considering $\left\{T_{I}: I \in I\right\}=\left\{T_{1}, T_{2}\right\}$ in Theorem 2.1, we have the required proof.

Remark 2.4. Corollary 2.3 extends and generalizes many existing fixed point theorems.

If we take $\psi(t)=t$ and $T_{i}=T$ in Theorem 2.1, we have the following corollary.

Corollary 2.5. Let $(X, p)$ be a complete partial metric space. Suppose $T: X \rightarrow X$ be a self mapping such that $T(X) \subseteq g(X), g X$ is closed in $(X, p)$ and for all $x, y \in X$

$$
p(T x, T y) \leq \varphi(M(x, y)),
$$

where $\varphi:[0, \infty) \rightarrow[0, \infty)$ is continuous, nondecreasing function such that $\phi(t)<t$ for each $t>0$ and

$$
M(x, y)=\max \left\{\begin{array}{l}
p(g x, g y), p(g x, T x), p(g y, T y), \\
\frac{p(g x, T y), p(g y, T x)}{2}
\end{array}\right\} .
$$

Then, there exists a unique $x \in X$ such that $g x=T x$. Moreover, we have $p(g x, g x)=0$.

If we take $g=I$ and $\varphi(t)=\lambda t$ for $\lambda \in[0,1)$ in Corollary 2.5, we have the following corollary.

Corollary 2.6. Let $(X, p)$ be a complete partial metric space. Suppose $T: X \rightarrow X$ be a self mapping and $g: X \rightarrow X$. Also $T(X) \subseteq g(X), g X$ is closed in $(X, p)$ and for all $x, y \in$ $X$

$$
p(T x, T y) \leq \lambda \max \left\{\begin{array}{l}
p(x, y), p(x, T x), p(y, T y), \\
\frac{p(x, T y), p(y, T x)}{2}
\end{array}\right\} .
$$

Then, there exists a unique $x \in X$ such that $x=T x$. Moreover, we have $p(x, x)=0$.

Now, we will prove the following result.

Theorem 2.7. Let $(X, p)$ be a complete partial metric space. Suppose $\left\{\mathrm{T}_{\mathrm{i}}: \mathrm{X} \rightarrow \mathrm{X}: \mathrm{i} \in \mathrm{I}\right\}$ be a family of mappings and $\mathrm{g}: \mathrm{X} \rightarrow \mathrm{X}$ be a self map. Also there exists $\mathrm{i}_{0} \in \mathrm{I}$ such that $T_{i_{0}}(\mathrm{X}) \subseteq \mathrm{g}(\mathrm{X})$ and

$$
\psi\left(p\left(T_{i_{0}} x, T_{i} y\right)\right) \leq \varphi(M(x, y)),
$$

where

$$
M(x, y)=\max \left\{\begin{array}{l}
p(g x, g y), p\left(g x, T_{i_{0}} x\right), p\left(g y, T_{i} y\right), \\
\frac{p\left(g x, T_{i} y\right), p\left(g y, T_{i_{0}} x\right)}{2}
\end{array}\right\},
$$


and $(\psi, \varphi) \in \Psi$. Also suppose $T_{i_{0}}, g$ are partial compatible and continuous in $(X, p)$. Then, there exists a unique $x \in X$ such that $g x=T_{i} x$ for all $i \in I$. Moreover, we have $p(g x, g x)=0$.

Proof. Using the same arguments in the proof of Theorem 2.1, we deduce that $\left\{T_{i_{0}}\left(x_{n}\right)\right\}$ is a Cauchy sequence in the complete metric space $\left(X, p^{s}\right)$, and therefore, there exists $z \in X$ such that

$$
\begin{aligned}
& p(z, z)=\lim _{n \rightarrow \infty} p\left(T_{i_{0}}\left(x_{n}\right), z\right)=\lim _{n \rightarrow \infty} p\left(g x_{n+1}, z\right) \\
& =\lim _{n, m \rightarrow \infty} p\left(T_{i_{0}}\left(x_{n}\right), T_{i_{0}}\left(x_{m}\right)\right)=0 .
\end{aligned}
$$

Now we show that $z$ is a coincidence point of $T_{i_{0}}$ and $g$.

Since $T_{i_{0}}$ and $g$ are continuous, from (2.24) and using Lemma 1.7, we get

$$
\begin{gathered}
p\left(T_{i_{0}} z, T_{i_{0}} z\right)=\lim _{n \rightarrow \infty} p\left(T_{i_{0}}\left(T_{i_{0}}\left(x_{n}\right)\right), T_{i_{0}} z\right), \\
p(g z, g z)=\lim _{n \rightarrow \infty} p\left(g\left(T_{i_{0}}\left(x_{n+1}\right)\right), g z\right),
\end{gathered}
$$

Since $T_{i_{0}}$ and $g$ are partial compatible mappings, this implies that

$$
\begin{aligned}
& p(g z, g z)=0 \\
& \text { and } \lim _{n \rightarrow \infty} p\left(g\left(T_{i_{0}}\left(x_{n+1}\right)\right), g\left(T_{i_{0}}\left(x_{n+1}\right)\right)\right)=0 .
\end{aligned}
$$

The condition $\left(p_{4}\right)$, we obtain

$$
\begin{aligned}
& p\left(T_{i_{0}} z, g z\right) \leq p\left(T_{i_{0}} z, T_{i_{0}}\left(T_{i_{0}}\left(x_{n}\right)\right)\right) \\
& +p\left(T_{i_{0}}\left(T_{i_{0}}\left(x_{n}\right)\right), g\left(T_{i_{0}}\left(x_{n+1}\right)\right)\right) \\
& +p\left(g\left(T_{i_{0}}\left(x_{n+1}\right)\right), g z\right) \\
& -p\left(T_{i_{0}}\left(T_{i_{0}}\left(x_{n}\right)\right), T_{i_{0}}\left(T_{i_{0}}\left(x_{n}\right)\right)\right) \\
& -p\left(g\left(T_{i_{0}}\left(x_{n+1}\right)\right), g\left(T_{i_{0}}\left(x_{n+1}\right)\right)\right) \\
& =p\left(T_{i_{0}} z, T_{i_{0}}\left(T_{i_{0}}\left(x_{n}\right)\right)\right) \\
& +p\left(T_{i_{0}}\left(g x_{n+1}\right), g\left(T_{i_{0}}\left(x_{n+1}\right)\right)\right) \\
& +p\left(g\left(T_{i_{0}}\left(x_{n+1}\right)\right), g z\right) \\
& -p\left(T_{i_{0}}\left(T_{i_{0}}\left(x_{n}\right)\right), T_{i_{0}}\left(T_{i_{0}}\left(x_{n}\right)\right)\right) \\
& -p\left(g\left(T_{i_{0}}\left(x_{n+1}\right)\right), g\left(T_{i_{0}}\left(x_{n+1}\right)\right)\right) .
\end{aligned}
$$

Letting $n \rightarrow \infty$ in the above inequality and using (2.24), (2.25) and (2.26), we have

$$
\begin{aligned}
p\left(T_{i_{0}} z, g z\right) & \leq p\left(T_{i_{0}} z, T_{i_{0}} z\right)+p(g z, g z) \\
& =p\left(T_{i_{0}} z, T_{i_{0}} z\right) .
\end{aligned}
$$

Now, we will prove that $p\left(T_{i_{0}} z, T_{i_{0}} z\right)=0$. Suppose that this is not the case. Then, from (2.22) with $i=i_{0}, y=x=z$, we get

$$
\psi\left(p\left(T_{i_{0}} z, T_{i_{0}} z\right)\right) \leq \varphi(M(z, z)),
$$

where

$$
\begin{aligned}
M(z, z) & =\max \left\{\begin{array}{l}
p(g z, g z), p\left(g z, T_{i_{0}} z\right), p\left(g z, T_{i_{0}} z\right), \\
\frac{p\left(g z, T_{i_{0}} z\right), p\left(g z, T_{i_{0}} z\right)}{2}
\end{array}\right\} \\
& =p\left(g z, T_{\alpha_{0}} z\right) .
\end{aligned}
$$

Therefore, from (2.27) and the above inequality, we have

$$
p\left(g z, T_{i_{0}} z\right)<p\left(g z, T_{i_{0}} z\right),
$$

a contradiction. Hence $p\left(g z, T_{i_{0}} z\right)=0$ which implies that $T_{i_{0}} z=g z$, that is, $z$ is a coincidence point of $T_{i_{0}}$ and $g$. If $w$ is a coincidence point of $T_{i_{0}}$ and $g$ other then $z$, then putting $i=i_{0}, x=z, y=w$ in (2.22) we have

$$
\psi\left(p\left(T_{i_{0}} z, T_{i_{0}} w\right)\right) \leq \varphi(M(z, w)),
$$

where

$$
\begin{aligned}
& M(z, w)=\max \left\{\begin{array}{l}
p(g z, g w), p\left(g z, T_{i_{0}} z\right), p\left(g w, T_{i_{0}} w\right), \\
\frac{p\left(g z, T_{i_{0}} w\right), p\left(g w, T_{i_{0}} z\right)}{2}
\end{array}\right\} \\
& =\max \left\{\begin{array}{l}
p\left(T_{i_{0}} z, T_{i_{0}} w\right), p\left(T_{i_{0}} z, T_{i_{0}} z\right), p\left(T_{\alpha_{0}} w, T_{\alpha_{0}} w\right), \\
\frac{p\left(T_{i_{0}} z, T_{i_{0}} w\right)+p\left(T_{i_{0}} w, T_{i_{0}} z\right)}{2}
\end{array}\right\} \\
& =p\left(T_{i_{0}} z, T_{i_{0}} w\right) .
\end{aligned}
$$

Therefore,

$$
\psi\left(p\left(T_{i_{0}} z, T_{i_{0}} w\right)\right) \leq \varphi\left(p\left(T_{i_{0}} z, T_{i_{0}} w\right)\right),
$$

which is possible only when $z=w$. Hence $z$ is the unique coincidence point of $T_{i_{0}}$ and $g$. Similar Theorem 2.1 can be shown that $z$ is a coincidence point of $\left\{T_{i}\right\}$ and $g$ for any $i \in I$.

We give in the following a sufficient condition for the uniqueness of the common fixed point of the mappings $\left\{T_{i}\right\}$ and $g$.

Theorem 2.8. Adding to the hypotheses of Theorem 2.1 (resp. Theorem 2.7) the condition:

$T_{i_{0}}$ and $g$ commute at their coincidence points, we obtain the uniqueness of common fixed point of $\left\{T_{i}\right\}$ and $g$.

Proof. Suppose that $T_{i_{0}}$ and $g$ commute at $x$. Set $y=$ $T_{i_{0}} x=g x$. Then

$$
T_{i_{0}} y=T_{i_{0}}(g x)=g\left(T_{i_{0}} x\right)=g y,
$$

from (2.1) we get

$$
\begin{aligned}
\psi\left(p\left(y, T_{i_{0}} y\right)\right) & =\psi\left(p\left(T_{i_{0}} x, T_{i_{0}} y\right)\right) \\
& \leq \psi(M(x, y)),
\end{aligned}
$$

where 


$$
\begin{aligned}
& M(x, y)=\max \left\{\begin{array}{l}
p(g x, g y), p\left(g x, T_{i_{0}} x\right), p\left(g y, T_{i_{0}} y\right), \\
\frac{p\left(g x, T_{i_{0}} y\right), p\left(g y, T_{i_{0}} x\right)}{2}
\end{array}\right\} \\
& =\max \left\{\begin{array}{l}
p\left(T_{i_{0}} x, T_{i_{0}} y\right), p\left(T_{i_{0}} x, T_{i_{0}} x\right), p\left(T_{i_{0}} y, T_{i_{0}} y\right), \\
\frac{p\left(T_{i_{0}} x, T_{i_{0}} y\right)+p\left(T_{i_{0}} y, T_{i_{0}} x\right)}{2}
\end{array}\right\} \\
& =p\left(T_{i_{0}} x, T_{i_{0}} y\right)=p\left(y, T_{i_{0}} y\right) .
\end{aligned}
$$

Suppose that $p\left(y, T_{i_{0}} y\right)>0$, from (2.29), we get

$$
\begin{aligned}
& \psi\left(p\left(y, T_{i_{0}} y\right)\right) \leq \varphi(M(x, y)) \\
& =\varphi\left(p\left(y, T_{i_{0}} y\right)\right)<\psi\left(p\left(y, T_{i_{0}} y\right)\right),
\end{aligned}
$$

which is a contradiction. Hence $p\left(y, T_{\alpha_{0}} y\right)=0$. Therefore,

$$
T_{i_{0}} y=g y=y .
$$

Thus we proved that $T_{i_{0}}$ and $g$ have a common fixed point.

Uniqueness: Let $v$ and $w$ be two common fixed points of $T_{i_{0}}$ and $g$. (i.e) $v=T_{i_{0}} v=g v$ and $w=T_{i_{0}} w=g w$. Using inequality (2.1), we have

$$
\psi\left(T_{i_{0}} w, T_{i_{0}} v\right) \leq \varphi(M(w, v)),
$$

where

$$
\begin{aligned}
M(w, v) & =\max \left\{\begin{array}{l}
p(g w, g v), p\left(g w, T_{i_{0}} w\right), p\left(g v, T_{i_{0}} v\right), \\
\frac{p\left(g w, T_{i_{0}} v\right)+p\left(g v, T_{i_{0}} w\right)}{2}
\end{array}\right\} \\
& =p(w, v) .
\end{aligned}
$$

Therefore,

$$
\psi(p(w, v))=\psi\left(p\left(T_{i_{0}} w, T_{i_{0}} v\right)\right) \leq \varphi(p(w, v))
$$

which is possible only when $w=v$. Hence $T_{i_{0}}$ and $g$ have an unique common fixed point. Now suppose that $x \in X$ is a common fixed point of $T_{i_{0}}$ and $g$. Then $x=g x=T_{i_{0}} x$.

For any $i \in I$, from inequality (2.1), we have

$$
\psi\left(p\left(x, T_{i} x\right)\right)=\psi\left(p\left(T_{i_{0}} x, T_{i} x\right)\right) \leq \varphi(M(x, x))(2.3
$$

where

$$
\begin{aligned}
& M(x, x)=\max \left\{\begin{array}{l}
p(g x, g x), p\left(g x, T_{i} x\right), p\left(g x, T_{i_{0}} x\right), \\
\frac{p\left(g x, T_{i} x\right)+p(x, x)}{2}
\end{array}\right\} \\
& =\max \left\{\begin{array}{l}
p(x, x), p\left(x, T_{i} x\right), p(x, x), \\
\frac{p\left(x, T_{i} x\right)+p(x, x)}{2}
\end{array}\right\} \\
& =p\left(x, T_{i} x\right)
\end{aligned}
$$

Suppose that $p\left(x, T_{i} x\right)>0$, from (2.31), we get

$$
\begin{aligned}
& \psi\left(p\left(x, T_{i} x\right)\right) \leq \varphi(M(x, x)) \\
& =\varphi\left(p\left(x, T_{i} x\right)\right)<\psi\left(p\left(x, T_{i} x\right)\right),
\end{aligned}
$$

which is a contradiction. Hence $p\left(x, T_{i} x\right)=0$. So, we have

$$
T_{i} x=x .
$$

that is, $x$ is a common fixed point of $g$ and $\left\{T_{i}: i \in I\right\}$.

Example 2.9. Let $X=[0,1]$ and $p(x, y)=\max \{x, y\}$, then it is clear that $(X, p)$ is a complete partial metric space. Let $I=\{1,2,3, \ldots\}, i_{0}=1$ and for every $i \in I, T_{i}, g: X \rightarrow X$, and $\psi, \varphi:[0, \infty) \rightarrow[0, \infty)$ be given by $T_{i}(x)=\frac{10}{i+2} x^{3}$, $g x=4 x^{3}, \psi(t)=4 t, \varphi(t)=\frac{10}{3} t$, Clearly $(\psi, \varphi) \in \Psi$. We show that condition (2.1) is satisfied.

If $x, y \in X$, then we have

$$
\begin{aligned}
& \psi\left(p\left(T_{i_{0}}(x), T_{i}(y)\right)\right)=\psi\left(p\left(T_{1}(x), T_{i}(y)\right)\right) \\
& =\psi\left(\max \left\{\frac{10}{3} x^{3}, \frac{10}{i+2} y^{3}\right\}\right) \\
& =4 \max \left\{\frac{10}{3} x^{3}, \frac{10}{i+2} y^{3}\right\} \\
& \leq \frac{4}{3} \max \left\{10 x^{3}, 10 y^{3}\right\}=\frac{10}{3} p(g x, g y) \\
& \leq \frac{10}{3} \max \left\{\frac{p(g x, g y), p\left(g x, T_{1}(x)\right), p\left(g y, T_{i}(y)\right),}{2}\right\} \\
& =\varphi(M(x, y)) .
\end{aligned}
$$

Note that, $\left\{T_{i}\right\}$ and $g$ satisfy all the conditions given in Theorem 2.7 and 2.8. Moreover, 0 is a unique common fixed point of $\left\{T_{i}\right\}$ and $g$.

\section{References}

[1] T. Abdeljawad, E. Karapinar, K. Tas, Existence and uniqueness of a common fixed point on partial metric spaces, Applied Mathematics Letters, 24(11)(2011),1900-1904.

[2] I. Altun, A. Erduran, Fixed point theorems for monotone mappings on partial metric spaces, Fixed Point Theory Appl 2011, 10.

[3] I. Altun, F. Sola, H. Simsek, Generalized contractions on Partial metric spaces, Topology and its Applications. 157(2010) 27782785.

[4] I. Altun, H. Simsek, Some fixed point theorems on dualistic partial metric spaces, J Adv Math Stud. 1, (2008) 1-8.

[5] I. Altun, A. Erduran, Fixed point theorems for monotone mappings on partial metric spaces, Fixed Point Theory Appl. 2011.

[6] M. Bukatin, R. Kopperman, S. Matthews, H. Pajoohesh, Partial metric spaces, Am. Math. Mon. 116,(2009) 708-718.

[7] E. Karapinar, M. E. Inci, Fixed point theorems for operators on partial metric spaces, Appl Math Lett 24(11)(2011) 1894-1899.

[8] S. G. Matthews, Partial metric topology, in: Proc. 8th Summer Conference on General Topology and Applications, in: Ann. New York Acad. Sci., vol. 728, 1994, pp. 183-197.

[9] S. Oltra, O. Valero, Banachs fixed point theorem for partial metric spaces, Rend. Istit. Mat. Univ. Trieste 36(2004)17-26.

[10] S. J. ONeill, Two topologies are better than one, Tech. report, University of Warwick, Coven-try, UK, http://www.dcs.warwick.ac.uk/reports/283.html, 1995.

[11] S. J. ONeill, Partial metrics, valuations and domain theory, in: Proc. 11th Summer Conference on General Topology and Applications, in: Ann. New York Acad. Sci., vol. 806, 1996, pp. 304-315. 
[12] S. Romaguera, A Kirk type characterization of completeness for partial metric spaces, Fixed Point Theory Appl. 2010(Ar-ticle ID 493298),6 (2010).

[13] S. Romaguera, O. Valero, A quantitative computational model for complete partial metric spaces via formal balls, Math. Structures Comput. Sci. 19 (3) (2009), 541-563.
[14] B. Samet, M. Rajovic, R. Lazovi, R. Stoiljkovic, Common Fixed Point Results For Nonlinear Contractions in Ordered Partial Metric Spaces, Fixed Point Theory Appl. 2011.

[15] O. Valero, On Banach fixed point theorems for partial metric spaces, Appl. Gen. Topol, 6 (2) (2005) 229-240. 